\title{
Clinicopathologic and Molecular Features of Mixed Neuroendocrine Non-Neuroendocrine Neoplasms of the Gallbladder
}

\author{
Mouyed Alawad $^{\text {a }}$, Raavi Gupta ${ }^{\text {a, b, d }}$, M.A. Haseeb ${ }^{\text {a, }}$, F. Charles Brunicardic
}

\begin{abstract}
Neuroendocrine neoplasms of the gallbladder are rare, comprising $0.5 \%$ of all neuroendocrine cancers and about $2 \%$ of gallbladder cancers. These neoplasms can also be found along with other malignant neoplasms of epithelial origin, mostly adenocarcinomas. Herein, we describe an unusual finding of a three-component mixed neuroendocrine non-neuroendocrine neoplasm (MiNEN) of the gallbladder. We also review the literature on 29 similar cases and summarize key features. We report on a 62-year-old woman who presented with right upper quadrant pain with a positive Murphy's sign. A clinical diagnosis of neoplasia was entertained and she underwent cholecystectomy. Gross examination of the specimen revealed a 5-cm exophytic mass at the gallbladder fundus. Histopathologic examination of the mass showed an infiltrating squamous cell carcinoma, an adjacent neuroendocrine carcinoma (each of these two components composed more than $30 \%$ of the neoplasm), and a superficial adenocarcinoma (composing $10 \%$ of the neoplasm). Gallbladder MiNENs present with similar symptoms and in the same age group as do carcinomas; however, their prognosis is often poor. Specific management and treatment guidelines have not been established since MiNENs are very rare.
\end{abstract}

Keywords: Gallbladder; Neuroendocrine tumors; Non-neuroendocrine tumors; MiNENs; Adenocarcinoma; Squamous cell carcinoma

\section{Introduction}

Gallbladder malignancies are rare in the United States but are

Manuscript submitted August 25, 2020, accepted October 23, 2020

Published online December 23, 2020

a Department of Pathology, State University of New York, Downstate Health Sciences University, Brooklyn, NY 11203, USA

bDepartment of Cell Biology, State University of New York, Downstate Health Sciences University, Brooklyn, NY 11203, USA

'Department of Surgery, State University of New York, Downstate Health Sciences University, Brooklyn, NY 11203, USA

${ }^{\mathrm{d} C o r r e s p o n d i n g ~ A u t h o r: ~ R a a v i ~ G u p t a, ~ D e p a r t m e n t ~ o f ~ P a t h o l o g y, ~ S t a t e ~ U n i-~}$ versity of New York, Downstate Health Sciences University, Brooklyn, NY 11203, USA. Email: raavi.gupta@downstate.edu

doi: https://doi.org/10.14740/gr1323 frequent in countries in Asia and Latin America. Adenocarcinomas comprise the vast majority of gallbladder cancers [1]. On the contrary, neuroendocrine neoplasms of the gallbladder comprise about $0.5 \%$ of all neuroendocine neoplasms, and about $2 \%$ of gallbladder cancers according to the Surveillance, Epidemiology, and End Results (SEER) registry [2]. Composite tumors, consisting of two or three of these histologic types, have also been described. Such tumors with multiple components also occur in the tubular gastrointestinal (GI) tract, along with pancreatobiliary tract and were used to be called mixed adenoneuroendocrine carcinomas (MANECs). To accommodate composite tumors with neuroendocrine and non-neuroendocrine components (adeno- and squamous cell carcinomas (SCC)) in the GI tract, the revised 2019 WHO classification has introduced a new subcategory called mixed neuroendocrine non-neuroendocrine neoplasms (MiNENs) [3]. It is noteworthy that such mixed tumors occur in multiple organ systems [4], and a consideration to create analogous categories for other systems is warranted since disease course for such tumors is not entirely understood.

MiNENs exhibit overlapping immunoreactivity in different components suggesting their origin from a common pluripotent cell [5]. This concept is further supported by molecular analyses of gallbladder small cell neuroendocrine carcinomas (NECs) which showed a range of mutations that substantially overlap with those seen in gallbladder adenocarcinomas [6]. Gallbladder NECs and MiNENs have a poor prognosis, and because of their rarity, specific treatment or management guidelines have not been established.

The MiNENs of the pancreas and tubular GI tract are characterized by the presence of at least $30 \%$ of each of the recognizable neuroendocrine and non-neuroendocrine components [3]. However, such a cutoff has not been defined for MiNENs in the gallbladder. Reporting and cataloguing composition of gallbladder MiNENs along with their disease course in all future cases would contribute to better understanding of these mixed tumors. To initiate cataloguing of mixed tumors of the gallbladder from the literature, we have identified wellcharacterized MiNENs with two and three components and have compiled their clinicopathological features and disease course.

Here we describe a patient who presented with right upper quadrant (RUQ) pain and underwent cholecystectomy. Histopathologic examination of the exophytic mass in the gallbladder revealed a neoplasm composed of three distinct carcino- 


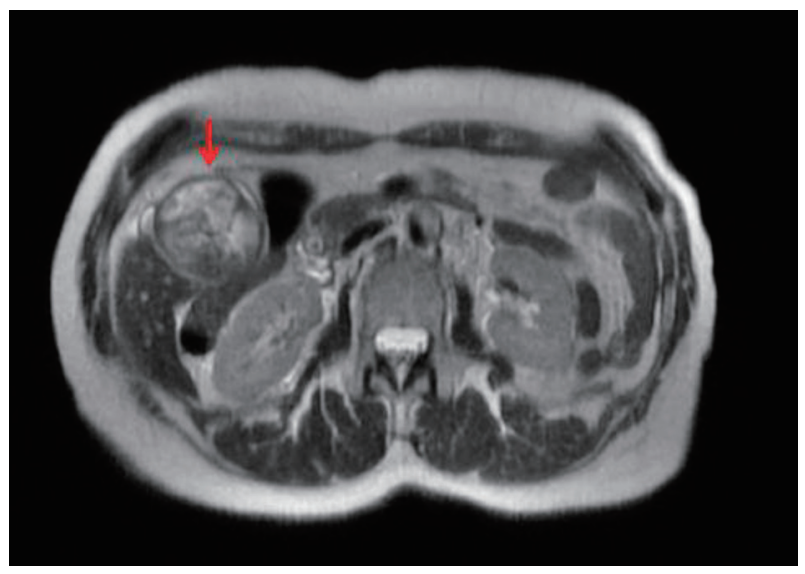

Figure 1. Magnetic resonance cholangiopancreatographic image showing a distended gallbladder (red arrow) with debris and stones (about $1 \mathrm{~cm}$ ) and two irregular septate nodules in the inferior fundus (about $1.5 \mathrm{~cm}$ ) that enhanced after gadolinium administration.

mas (SCC, adenocarcinoma and small cell NEC) which met the definition of MiNEN.

\section{Case Report}

A 62-year-old African-American woman presented to the emergency department with a 3-day history of RUQ pain, decreased oral intake, and non-bloody, non-bilious emesis. Vital signs were within normal limits, and physical examination was notable for moderate tenderness in the RUQ with a positive Murphy's sign. There was no guarding, rebound tenderness, nor hepatomegaly. Past medical history was significant for hypertension, hyperlipidemia and diabetes mellitus. Laboratory results were notable for total bilirubin of $5.80 \mathrm{mg} / \mathrm{dL}$ (reference: $0.30-1.00 \mathrm{mg} / \mathrm{dL}$ ), alkaline phosphatase of $426 \mathrm{U} / \mathrm{L}$ (reference: $34-104 \mathrm{U} / \mathrm{L}$ ), aspartate aminotransferase (AST) of $663 \mathrm{U} / \mathrm{L}$ (reference: 13 - $39 \mathrm{U} / \mathrm{L}$ ) and alanine aminotransferase (ALT) of $703 \mathrm{U} / \mathrm{L}$ (reference: 7 - $52 \mathrm{U} / \mathrm{L}$ ). Lipase was within the reference range. Basic metabolic panel (BMP) was remarkable only for glucose of $343 \mathrm{mg} / \mathrm{dL}$ (reference: 70 - 99 $\mathrm{mg} / \mathrm{dL}$ ). Hemogram parameters were within their respective reference ranges, and there was no leukocytosis. Lactate was $3.0 \mathrm{mmol} / \mathrm{L}$ (reference: $2-4 \mathrm{mmol} / \mathrm{L}$ ), and the patient was markedly alkalotic with a venous $\mathrm{pH}$ of 7.62. An ultrasound of the RUQ revealed a distended gallbladder with heterogeneous echogenicity of unclear etiology, along with intrahepatic and common bile duct dilatation.

Upon admission, a magnetic resonance cholangiopancreatography revealed debris and stones (about $1 \mathrm{~cm}$ ) and two irregular septate nodules (about $1.5 \mathrm{~cm}$ ) in the inferior fundus of the gallbladder (Fig. 1). Multiple gallstones were also seen. The common bile duct measured $11 \mathrm{~mm}$ in diameter and was noted to be filled with debris. Endoscopic retrograde cholangiopancreatography revealed thick sludge obstructing the ampulla of Vater and was extracted with a balloon sweep, followed by placement of a stent in the common bile duct. On hospital day 7, a laparoscopic cholecystectomy was started and

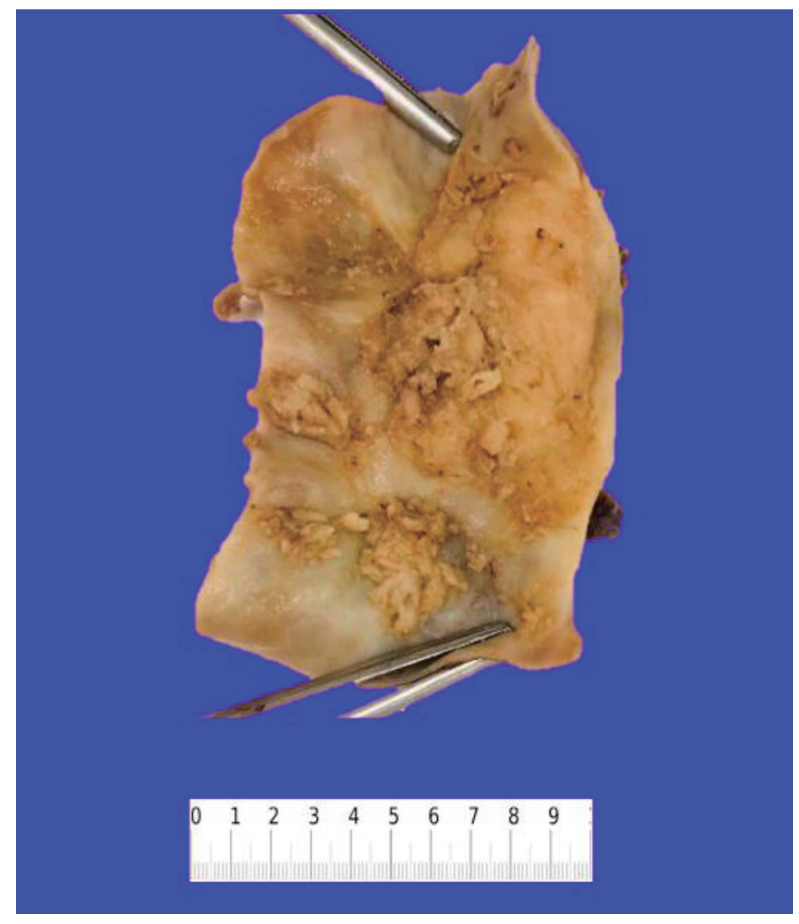

Figure 2. Resected gallbladder specimen showing multiple exophytic masses in the lumen.

was converted intraoperatively into an open cholecystectomy due to severe gallbladder distention. The post-operative course was uneventful, with gradually resolving abdominal pain, and the patient was discharged the following day.

Gross examination of the resected specimen showed an exophytic mass $(5 \times 4 \times 2 \mathrm{~cm})$ located at the fundus of the gallbladder (Fig. 2). Histopathologic examination of the mass showed a neoplasm consisting of three distinct components. An infiltrating SCC (60\%) and an adjacent NEC (30\%) were observed (Fig. 3a). The squamous component showed moderate differentiation with areas of keratinization (Fig. 3b). The neuroendocrine component showed areas of necrosis; the neuroendocrine cells had moderate cytoplasm, inconspicuous nucleoli and "salt and pepper" chromatin, with numerous mitotic figures (Fig. 3c). A third adenocarcinoma component comprised about $10 \%$ of the neoplasm (Fig. 3d). The tumor infiltrated the muscularis propria into perimuscular connective tissue.

The SCC was positive for p40 (Fig. 4a), p63 and CK7; the neuroendocrine component was positive for synaptophysin (Fig. 4b) and chromogranin; and the adenocarcinoma component was positive for CK7 (Fig. 4c) and mucin by mucicarmine, but negative for $\mathrm{p} 40$ and $\mathrm{p} 63$.

Because of these findings, the patient was readmitted for a partial hepatectomy of segments IVb and V of the liver along with lymph node dissection for staging. Histopathologic review of the partial hepatectomy, perihepatic lymph nodes and portal lymph nodes was negative for malignancy; the patient's tumor was classified as stage IIA. The patient was started on eight cycles of leucovorin, oxaliplatin and 5-fluorouracil (5FU).

Three months later, a positron emission tomography 


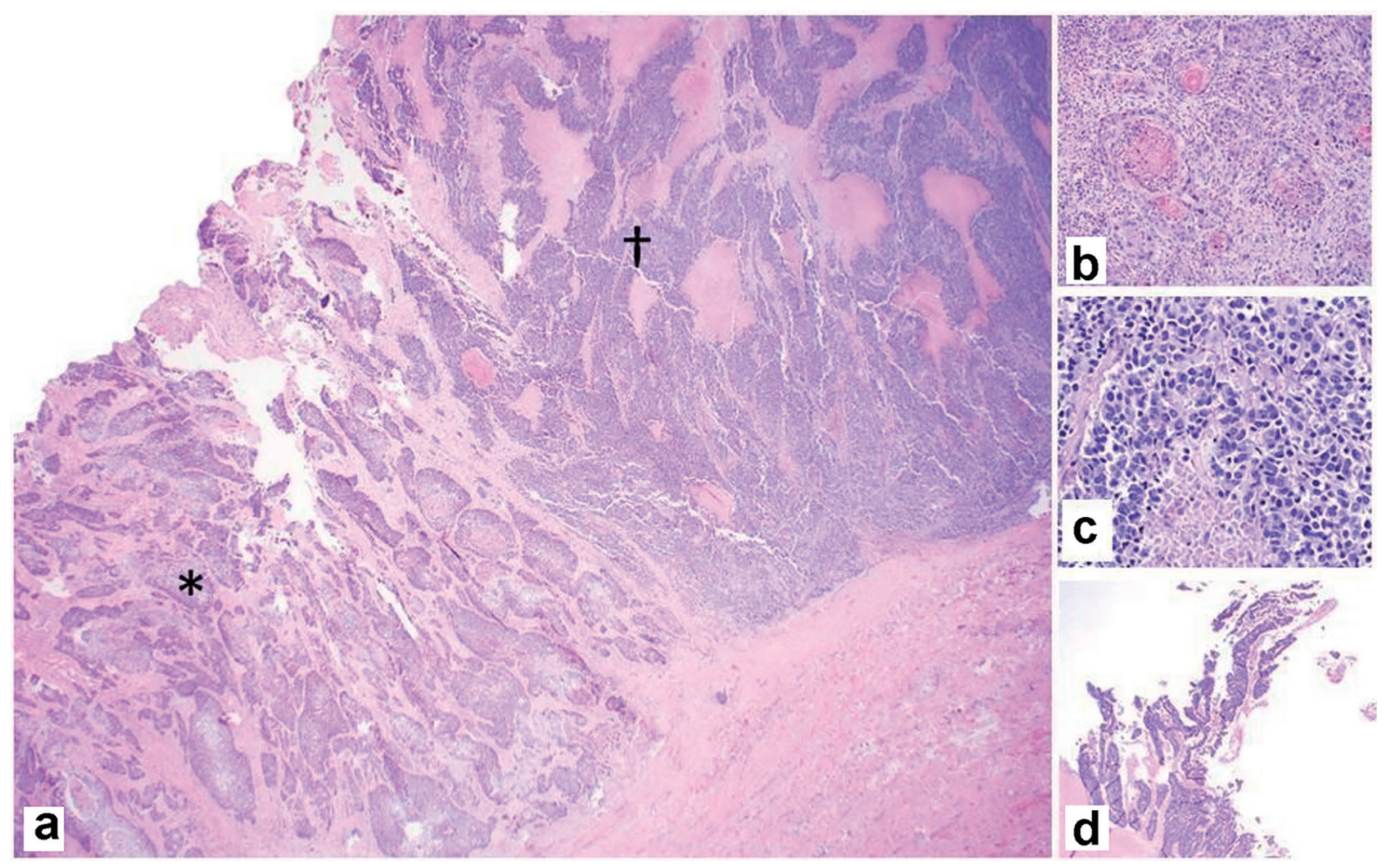

Figure 3. Representative photomicrographs of the MiNEN. (a) Infiltrating squamous cell carcinoma $\left(^{*}\right)$ and neuroendocrine carcinoma $(\dagger)$ adjacent to each other $(\times 20)$. (b) Squamous component with keratinization $(\times 100)$. (c) Neuroendocrine component with necrosis ( $\times 200)$. (d) Adenocarcinoma component with gland formation ( $\times 20)$. H\&E stain. MiNEN: mixed neuroendocrine non-endocrine neoplasm; H\&E: hematoxylin and eosin.

(PET) scan revealed hypermetabolic enlarged portacaval lymphadenopathy and a hypermetabolic soft tissue nodule in the anterior abdomen to the left of the umbilicus, suggesting meta- static disease. Biopsy of the nodule showed a poorly differentiated carcinoma that was positive for p63 and negative for synaptophysin supporting squamous differentiation and consistent

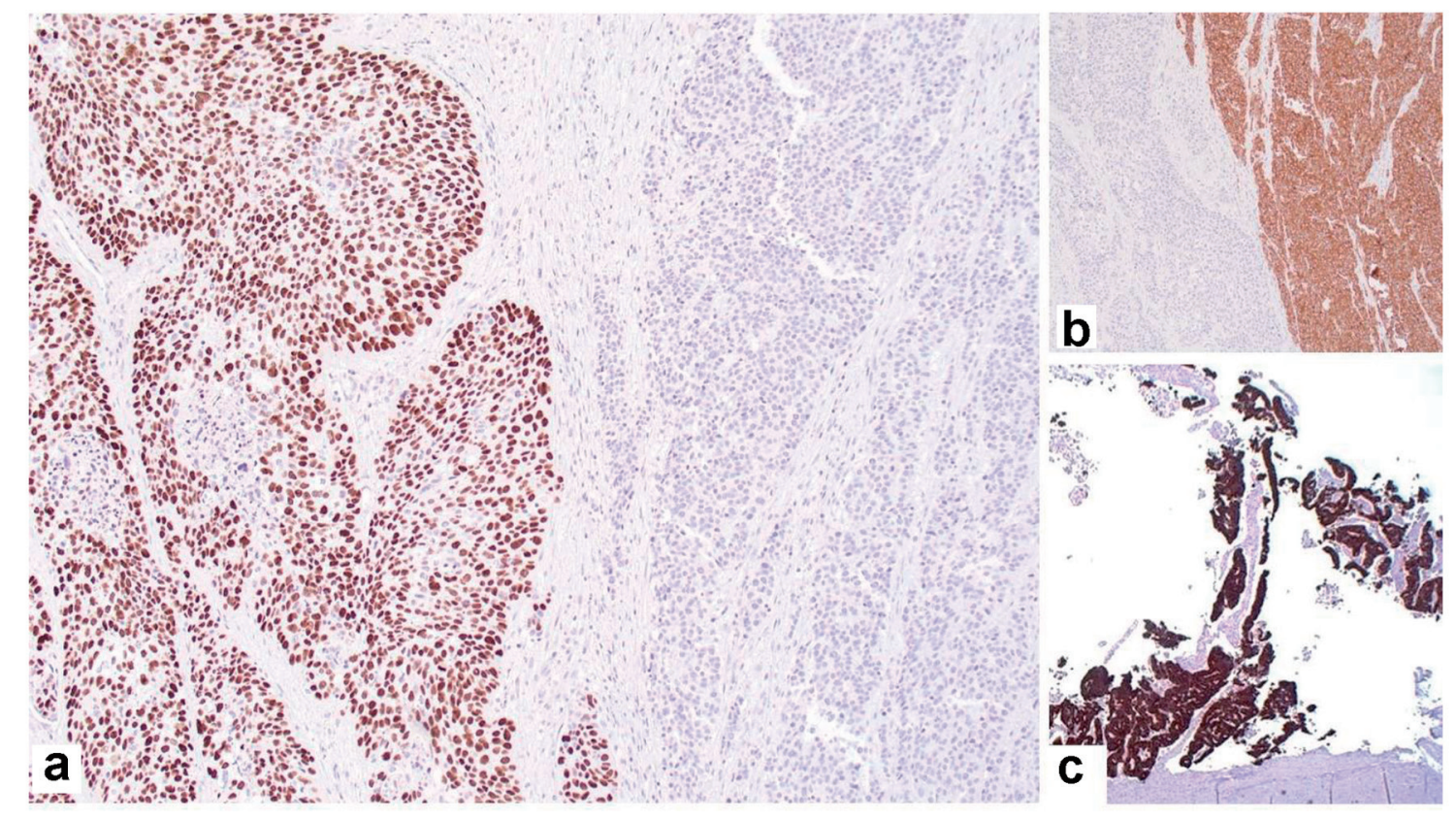

Figure 4. Immunohistochemical staining showing: (a) p63-positive squamous component and negative neuroendocrine component (× 40); (b) synaptophysin-positive neuroendocrine component and negative squamous cell component ( $\times 40)$; (c) CK7positive adenocarcinoma component $(\times 20)$. 


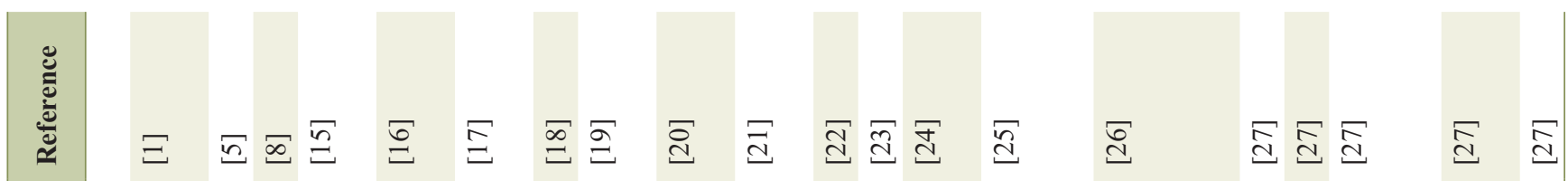

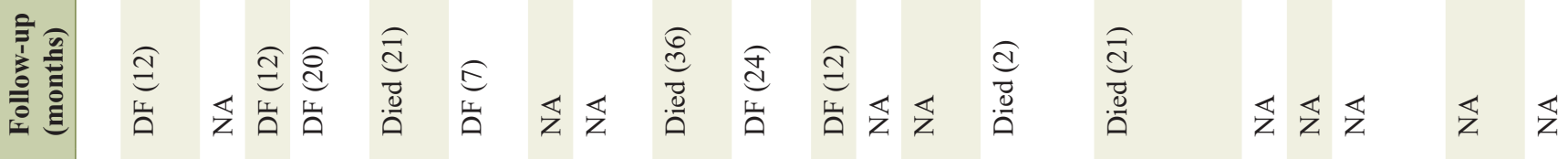

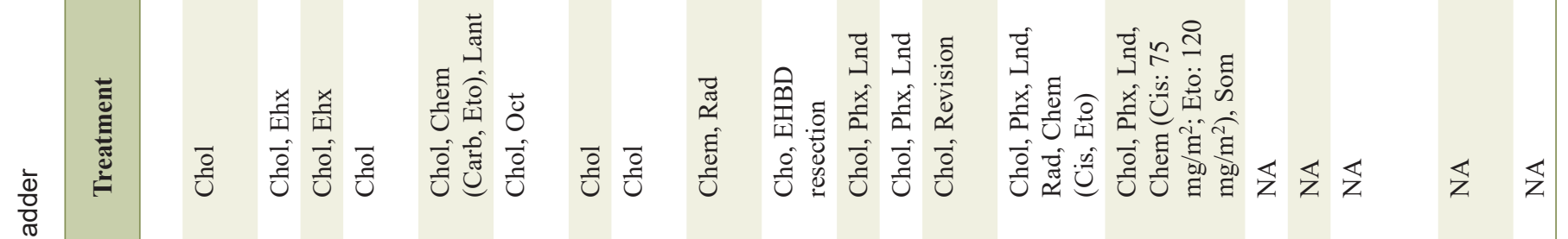

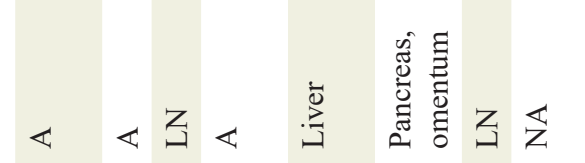
Ż

$\stackrel{\infty}{\frac{0}{2}}$ <

(5) $=$ $\begin{array}{ll}0 \\ ن & 0 \\ 0 & 0 \\ 0 & 0\end{array}$ 岱岱

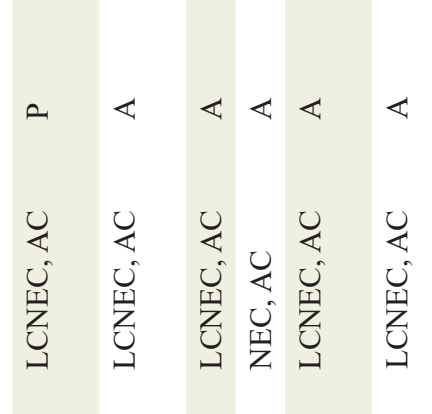

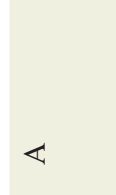

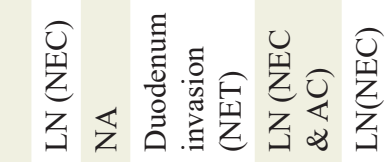

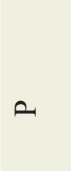

这

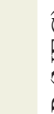

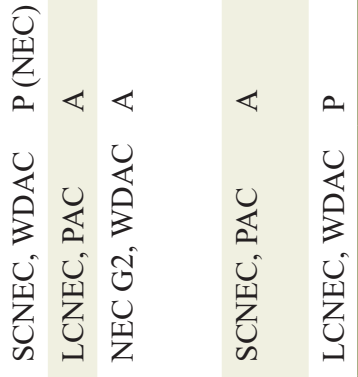

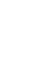

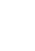

政

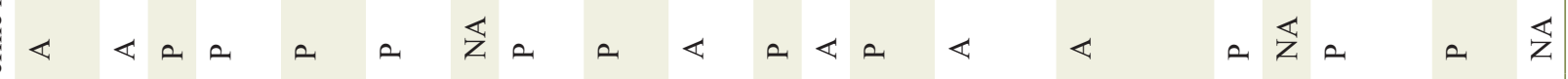

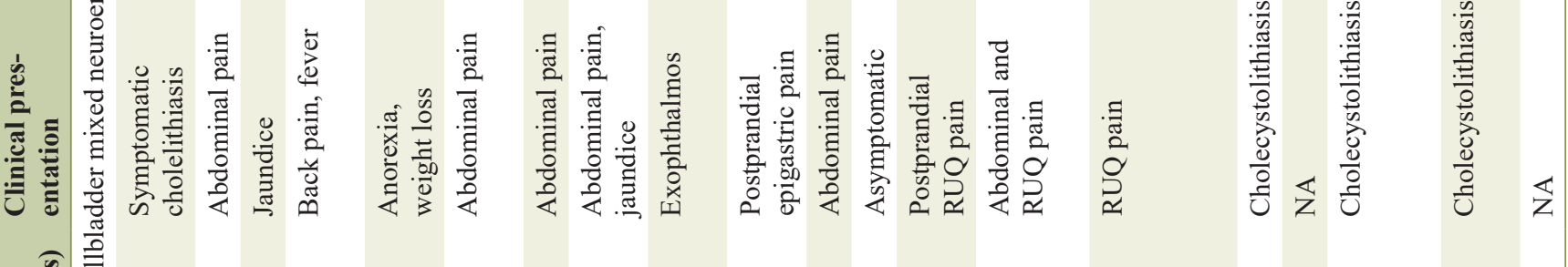

$\stackrel{\infty}{\infty} \stackrel{\infty}{i} \stackrel{\infty}{=}$ $\sum^{\infty} \frac{n}{n}$

$\stackrel{i n}{\sum} \stackrel{i}{\Sigma}$

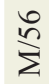
$\frac{\mathrm{N}}{\mathrm{N}}$

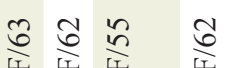

$\frac{\sqrt{2}}{2}$

$\begin{array}{llll}\frac{1}{1} & \frac{1}{5} & \frac{\pi}{4} & \frac{1}{4}\end{array}$

$\stackrel{\sim}{=} \stackrel{\infty}{-}$

2 ก 


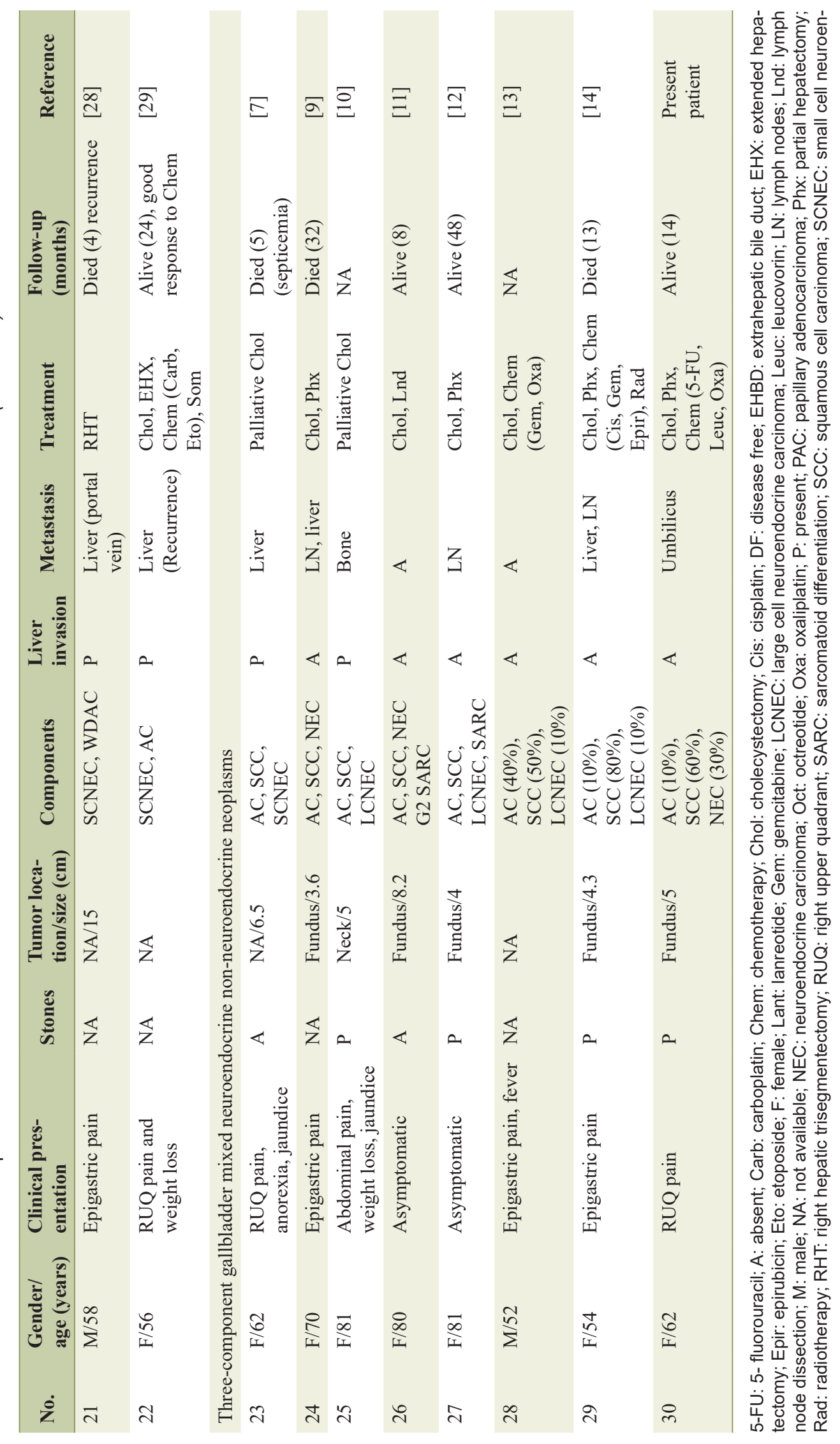




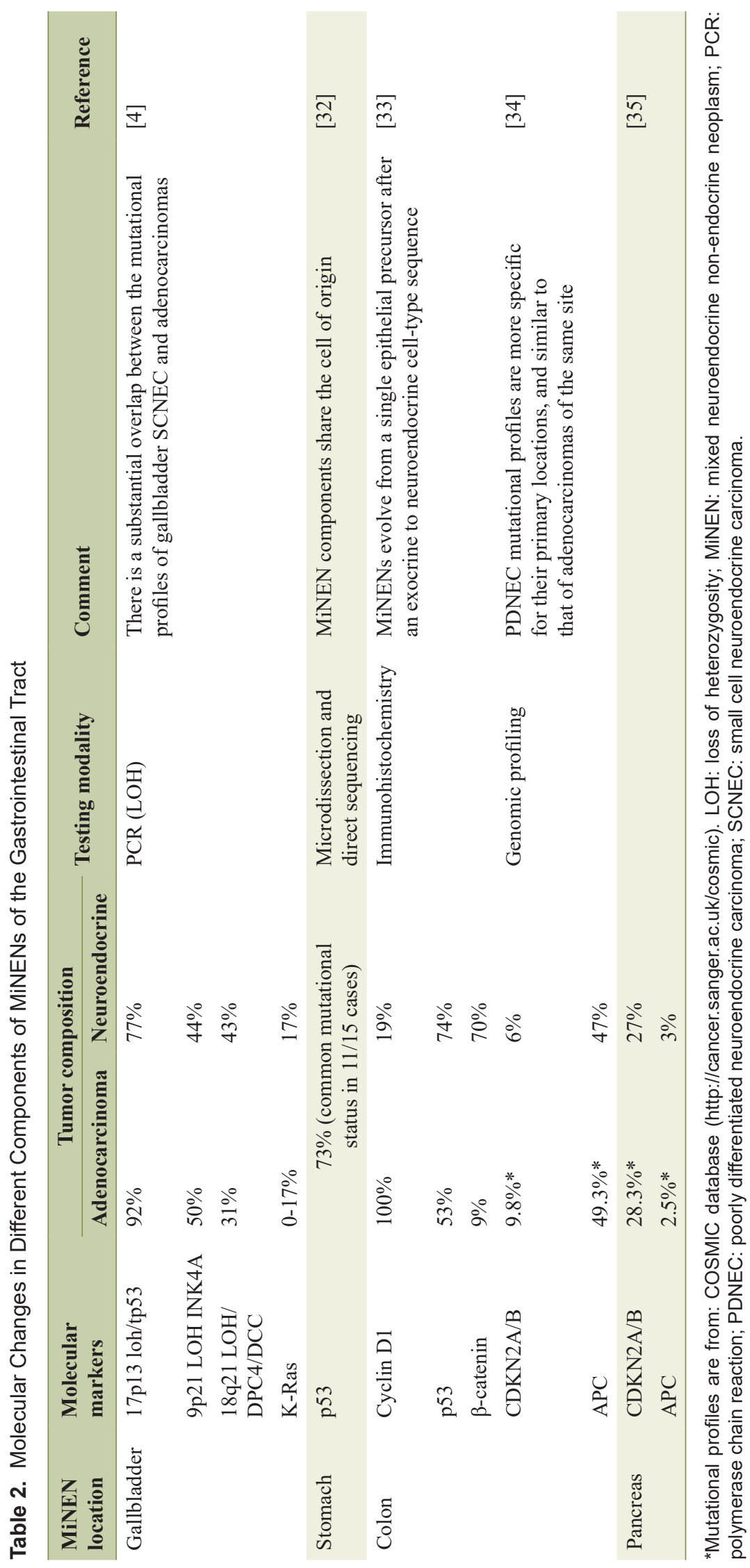


with metastasis.

The patient is alive and well at 14 months after the initial diagnosis.

\section{Clinicopathologic features of MiNENs}

Since the behavior of gallbladder tumors with mixed neuroendocrine and non-neuroendocrine components is not well documented, we reviewed the literature for these mixed tumors (two or three components). We searched PubMed and Google Scholar for "mixed gallbladder tumor", "mixed gallbladder carcinoma", "composite gallbladder tumor", "composite gall bladder carcinoma", "adenocarcinoma of the gallbladder", "squamous cell carcinoma of the gall bladder", "neuroendocrine carcinoma of the gallbladder" and "neuroendocrine tumor of the gall bladder". We found 15 cases of mixed gallbladder neoplasms with two-component (NEC and adenocarcinoma) and eight cases with three-component (NEC, SCC and adenocarcinoma) neoplasms (Table 1 [1, 5, 7-29]).

The patients with two-component tumors presented at a mean age of 58 years (range: 45 - 85 years) and the male/female ratio was 1:2. Most of the patients presented with generalized abdominal pain and some with pain localized in the RUQ. Other symptoms included jaundice $(2 / 22)$, fever $(1 / 22)$ and weight loss $(2 / 22)$. Gallstones were documented in $11 / 22$ patients. The mean size of the tumor was $6 \mathrm{~cm}$ (range: $<1-$ $15 \mathrm{~cm}$ ); it was located in the gallbladder fundus in $7 / 22$, and the body in $3 / 22$ patients. In four patients, the tumor occupied more than one anatomic region of the gallbladder, and in four patients, the tumor extended beyond the gallbladder to involve the liver, pancreas or duodenum. The NEC component of the tumor was composed of large cell NEC in $13 / 22$ patients and of small cell NEC in $6 / 22$ patients. One patient had both large cell and small cell NECs, and another had a grade 2 NEC. Five patients presented with metastasis to regional lymph nodes and another three to other viscera. In one series of cases, three patients had metastasis of only the neuroendocrine component, and one patient had metastasis of both the neuroendocrine and adenocarcinoma components. The adenocarcinoma was classified as well differentiated in four patients, and as papillary adenocarcinoma in two patients [27]. Cholecystectomy was performed in 14/22 patients with additional partial hepatectomy in $8 / 22$ patients, and chemotherapy in five of these. A combination of carboplatin and etoposide was used in two patients and a combination of cisplatin and etoposide in another two. Details of chemotherapy for the fifth patient are not available. A somatostatin analogue was used in three of the five patients on chemotherapy. The patients without liver invasion or metastases were disease-free for a mean of 14.5 months (range: 7 - 24 months), and were alive at the reporting time. Of the five patients who died, three survived for a mean of 26 months (range: 21 - 36 months).

The patients with three-component tumors presented at a mean age of 68 years (range: 52 - 81 years) and the male/ female ratio was $1: 3$. The patients presented with epigastric (3/8), RUQ (2/8) and abdominal (1/8) pain; two patients were asymptomatic. Two of eight patients presented with anorexia or weight loss. Gallstones were documented in $5 / 8$ patients. The mean size of tumor was $5.2 \mathrm{~cm}$ (range: $3.6-8.2 \mathrm{~cm}$ ), and was located in the fundus in $5 / 8$, and in the gallbladder neck in $1 / 8$ patients. The tumor was composed of large cell NEC in $4 / 8$, small cell NEC in $1 / 8$ and unspecified NEC in $2 / 8$ patients. Sarcomatoid differentiation was observed in two patients. The proportion of tumor components was reported in only three patients (including the patient presented here). All three patients had $\geq 50 \%$ of the tumor composed of SCC, and neuroendocrine component comprised $10-30 \%$ of the tumor. One patient presented with metastasis only to regional lymph nodes, 2/8 presented with direct liver invasion, $3 / 8$ presented with liver metastasis and two presented with distant metastasis to the umbilicus or bone. Three of the eight patients received chemotherapy; one was treated with oxaliplatin and gemcitabine and another with cisplatin, gemcitabine and epirubicin. Our patient was given leucovorin, oxaliplatin and 5-FU. The patients with tumors localized in the gallbladder and those with only regional lymph node metastasis were disease-free for 8 and 48 months, respectively. The four patients who died between 5 and 32 months (mean: 14 months) all had metastasis.

\section{Origin and genetics of MiNENs}

The origin of gallbladder neuroendocrine neoplasms is enigmatic. Neuroendocrine tumors of the tubular gut most often occur in the rectum, appendix and ileum where they arise from hormone-producing cells known as amine precursor uptake and decarboxylation (APUD) cells. However, normal gallbladder mucosa lacks APUD cells. At least two different hypotheses have been proposed to explain the origin of neuroendocrine tumors of the gallbladder: these arise from the epithelial metaplasia of the gallbladder which includes goblet cells and enterochromaffin cells [30]; and from pluripotent stem cells. This is based on demonstration of shared immunoreactivity patterns between different tumor components and common characteristics (featuring both neuroendocrine and glandular differentiation) observed in electron micrographs [5, 7, 8, 30, 31].

Gallbladder small cell NECs have molecular abnormalities that overlap with those observed in gallbladder adenocarcinomas. Molecular studies of 12 gallbladder small cell NECs revealed loss of heterozygosity (LOH) at 16p13 (TP53 locus) in $77 \%$ of the cases, a finding also observed in $92 \%$ of gallbladder adenocarcinomas. Similarly, LOH at 9p21 (INK4a locus) and 18q21 (DCC and DPC416 loci) found in 44\% and $43 \%$ of small cell NEC cases were similar to those reported in gallbladder adenocarcinomas (50\% and 31\%, respectively). KRAS mutation, found in $17 \%$ of small cell NEC cases, is considered a low frequency $(0-17 \%)$ in gallbladder adenocarcinomas. However, studies from Japan reported a higher frequency of KRAS mutation in gallbladder adenocarcinomas [6] (Table 2 [4, 32-35]).

Molecular studies showed that CDKN2A/B and APC mutations were present in $27 \%$ and $3 \%$ of pancreatic NECs, respectively, a pattern similar to that of pancreatic adenocarcinomas where the mutations were present in $28.3 \%$ and $2.5 \%$, respectively. Similarly, CDKN2A/B and APC mutations were 
present in $6 \%$ and $47 \%$ of colon NECs, in comparison to $9.8 \%$ and $49.3 \%$ of colon adenocarcinomas, respectively (Table 2) $[32,33]$.

\section{Discussion}

We have described a patient with a rare gallbladder tumor that consisted of neuroendocrine (small cell carcinoma) and nonneuroendocrine (adenocarcinoma and SCC) components. Because of rarity of gallbladder MiNENs, their clinicopathologic data are sparse, and we considered it useful to review the literature and summarize the reported findings.

Carcinomas of the gallbladder are most often of one histologic type. Of these, adenocarcinomas are the most common followed by NEC and SCC. Mixed tumors with two and three components have been reported in the GI tract including the gallbladder. Patients with one-, two- or three-component gallbladder tumors most often present in their sixth and seventh decades of life [36]. Most patients present with vague abdominal pain which is usually associated with cholelithiasis. NECs, when present, may involve extrahepatic bile ducts in addition to the body of the gallbladder, and patients may have additional symptoms of abdominal distension, ascites, jaundice and weight loss and. Isolated NECs with both large cell and small cell components have been reported, and more than one-third of these included an adenocarcinoma component. The striking feature of NECs in MiNENs is the predominance of large cell component which was distinct from predominance of small cell component in pure NECs [37].

The prognosis of MiNENs with two or three components is poor and similar to that of SCC and NEC, in contrast to adenocarcinomas of the gallbladder. Patients were followed up for $\leq 5$ years and those with metastasis died within 3 years of diagnosis. Metastasis at presentation or shortly after resection was a common finding in MiNENs. Metastasis was observed with greater frequency in patients with three-component than in those with two-component tumors, and so was the frequency of death. Although neuroendocrine component is expected to metastasize, it is notable that only the squamous component was identified in the metastatic lesion in our case. This feature of potential prognostic significance has not been documented in any of the cases we reviewed.

Staging of all gallbladder carcinomas is based on the depth of gallbladder wall invasion, and is of prognostic significance. However, we suggest that in addition to the depth of invasion, the quantification of each tumor subtype in MiNENs should also be reported. It will help better outline the prognosis of that tumor as each component is correlated with a distinct metastatic potential. Patients with adenocarcinoma of the gallbladder have a 5 -year survival of $45-70 \%$, followed by NEC $(20 \%)$ and SCC [3]. Most of the case reports we reviewed did not include proportion of subtypes; we believe this added information would help WHO revise its definition of gallbladder MiNENs in a manner similar to those of tubular GI. The origin of these tumors from gallbladder progenitor cells is under investigation, and further molecular studies are expected to help understand the biology of these tumors.

\section{Acknowledgments}

None to declare.

\section{Financial Disclosure}

No specific funding was used for this study.

\section{Conflict of Interest}

The authors declare no potential conflict of interest.

\section{Informed Consent}

The Institution Review Board and Privacy Board determined that this project does not meet the definition of human subject research (IRB-1639814-1).

\section{Author Contributions}

MA and RG made the diagnosis and wrote the first draft; MAH conceptualized and edited the manuscript; CFB supervised and guided the study, and edited the manuscript.

\section{Data Availability}

Any inquiries regarding supporting data availability of this study should be directed to the corresponding author.

\section{Abbreviations}

ALT: alanine aminotransferase; AST: aspartate aminotransferase; BMP: basic metabolic panel; PET: positron emission tomography; NEC: neuroendocrine carcinoma; RUQ: right upper quadrant; SCC: squamous cell carcinoma

\section{References}

1. Papotti M, Cassoni P, Sapino A, Passarino G, Krueger JE, Albores-Saavedra J. Large cell neuroendocrine carcinoma of the gallbladder: report of two cases. Am J Surg Pathol. 2000;24(10):1424-1428.

2. Yao JC, Hassan M, Phan A, Dagohoy C, Leary C, Mares JE, Abdalla EK, et al. One hundred years after "carcinoid": epidemiology of and prognostic factors for neuroendocrine tumors in 35,825 cases in the United States. J Clin Oncol. 2008;26(18):3063-3072.

3. WHO Classification of Tumours Editorial Board. Digestive System Tumours. WHO Classification of Tumours, 5th Ed. Lyon, France. International Agency for Research 
on Cancer, 2019.

4. La Rosa S, Sessa F, Uccella S. Mixed neuroendocrinenonneuroendocrine neoplasms (MiNENs): unifying the concept of a heterogeneous group of neoplasms. Endocr Pathol. 2016;27(4):284-311.

5. Paniz Mondolfi AE, Slova D, Fan W, Attiyeh FF, Afthinos J, Reidy J, Pang Y, et al. Mixed adenoneuroendocrine carcinoma (MANEC) of the gallbladder: a possible stem cell tumor? Pathol Int. 2011;61(10):608-614.

6. Maitra A, Tascilar M, Hruban RH, Offerhaus GJ, Albores-Saavedra J. Small cell carcinoma of the gallbladder: a clinicopathologic, immunohistochemical, and molecular pathology study of 12 cases. Am J Surg Pathol. 2001;25(5):595-601.

7. Iida Y, Tsutsumi Y. Small cell (endocrine cell) carcinoma of the gallbladder with squamous and adenocarcinomatous components. Acta Pathol Jpn. 1992;42(2):119-125.

8. Sato K, Imai T, Shirota Y, Ueda Y, Katsuda S. Combined large cell neuroendocrine carcinoma and adenocarcinoma of the gallbladder. Pathol Res Pract. 2010;206(6):397-400.

9. Mori M, Sato T, Yamamoto K, Seki H, Ishikawa H, Tsutsumi Y. A case of adenoendocrine cell carcinoma of the gallbladder with squamous differentiation in metastatic lymph node. Jpn J Gastroenterol Surg. 2005;38:63-68.

10. Noske A, Pahl S. Combined adenosquamous and largecell neuroendocrine carcinoma of the gallbladder. Virchows Arch. 2006;449(1):135-136.

11. Shintaku M, Kataoka K, Kawabata K. Mixed adenoneuroendocrine carcinoma of the gallbladder with squamous cell carcinomatous and osteosarcomatous differentiation: report of a case. Pathol Int. 2013;63(2):113-119.

12. Abe T, Kajiyama K, Harimoto N, Gion T, Shirabe K, Nagaie T. Composite adeno-endocrine carcinoma of the gallbladder with long-term survival. Int J Surg Case Rep. 2013;4(5):504-507.

13. Chen X-S, Xie X-F, Zhou J-J. Mixed large cell neuroendocrine carcinoma and adenosquamous carcinoma of gallbladder: a case report. Int J Clin Exp Pathol. 2017;10:1882-1885.

14. Jung J, Chae YS, Kim CH, Lee Y, Lee JH, Kim DS, Yu YD, et al. Combined adenosquamous and large cell neuroendocrine carcinoma of the gallbladder. J Pathol Transl Med. 2018;52(2):121-125.

15. Oshiro $H$, Matsuo $K$, Mawatari $H$, Inayama $Y$, Yamanaka S, Nagahama K, Endo I, et al. Mucin-producing gallbladder adenocarcinoma with focal small cell and large cell neuroendocrine differentiation associated with pancreaticobiliary maljunction. Pathol Int. 2008;58(12):780-786.

16. Iype S, Mirza TA, Propper DJ, Bhattacharya S, Feakins RM, Kocher HM. Neuroendocrine tumours of the gallbladder: three cases and a review of the literature. Postgrad Med J. 2009;85(1002):213-218.

17. Song W, Chen W, Zhang S, Peng J, He Y. Successful treatment of gallbladder mixed adenoneuroendocrine carcinoma with neo-adjuvant chemotherapy. Diagn Pathol. 2012;7:163.

18. Russo S, Russo F, Maiello FM, Paolini B, Carrabba A, De Gregorio A. Biphasic large cell neuroendocrine carcinoma-pure mucinous carcinoma of the gallblad- der (MANEC): a unique combination. Pathologica. 2012;104(4):185-189.

19. Al-Brahim N, Albannai R. Combined large cell neuroendocrine carcinoma and adenocarcinoma of the gallbladder. Endocr Pathol. 2013;24(2):110-113.

20. Nakagawa T, Sakashita N, Ohnishi K, Komohara Y, Takeya M. Imprint cytological feature of large cell neuroendocrine carcinoma of the gallbladder: a case report. J Med Invest. 2013;60(1-2):149-153.

21. Meguro Y, Fukushima N, Koizumi M, Kasahara N, Hydo M, Morishima K, Sata N, et al. A case of mixed adenoneuroendocrine carcinoma of the gallbladder arising from an intracystic papillary neoplasm associated with pancreaticobiliary maljunction. Pathol Int. 2014;64(9):465-471.

22. Liu W, Wang L, He XD, Feng C, Chang XY, Lu ZH. Mixed large cell neuroendocrine carcinoma and adenocarcinoma of the gallbladder: a case report and brief review of the literature. World J Surg Oncol. 2015;13:114.

23. Azad S, Shukla D, Garg A, Negi SS, Malhotra V. Mixed adenoneuroendocrine carcinoma of the gallbladder, histopathological features. Indian J Pathol Microbiol. 2015;58(4):543-545.

24. Acosta AM, Hamedani FS, Kajdacsy-Balla A, Wiley EL. Primary Mixed Adenoneuroendocrine Carcinoma of the Gallbladder in a 55-Year-Old Female Patient: A Case Report and Review of the Literature. Int J Surg Pathol. 2015;23(5):414-418.

25. Soin S, Pannu BS, Myint PT, Dhillon AS. Large cell neuroendocrine carcinoma and adenocarcinoma of gallbladder with concomitant hepatitis $\mathrm{C}$ infection. BMJ Case Rep. 2018;2018:bcr-2018-225141.

26. Lin YX, Jia QB, Fu YY, Cheng NS. Mixed Adenoneuroendocrine Carcinoma of the Gallbladder. J Gastrointest Surg. 2018;22(8):1452-1454.

27. Harada K, Sato Y, Ikeda H, Maylee H, Igarashi S, Okamura A, Masuda S, et al. Clinicopathologic study of mixed adenoneuroendocrine carcinomas of hepatobiliary organs. Virchows Arch. 2012;460(3):281-289.

28. Shimizu T, Tajiri T, Akimaru K, Arima Y, Yoshida H, Yokomuro S, Mamada Y, et al. Combined neuroendocrine cell carcinoma and adenocarcinoma of the gallbladder: report of a case. J Nippon Med Sch. 2006;73(2):101-105.

29. Skalicky A, Vistejnova L, Dubova M, Malkus T, Skalicky T, Troup O. Mixed neuroendocrine-non-neuroendocrine carcinoma of gallbladder: case report. World J Surg Oncol. 2019;17(1):55.

30. Laitio M. Goblet cells, enterochromaffin cells, superficial gastric-type epithelium and antral-type glands in the gallbladder. Beitr Pathol. 1975;156(4):343-358.

31. Harada K, Sato Y, Ikeda H, Hsu M, Igarashi S, Nakanuma Y. Notch1-Hes1 signalling axis in the tumourigenesis of biliary neuroendocrine tumours. J Clin Pathol. 2013;66(5):386-391.

32. Nishikura K, Watanabe H, Iwafuchi M, Fujiwara T, Kojima K, Ajioka Y. Carcinogenesis of gastric endocrine cell carcinoma: analysis of histopathology and p53 gene alteration. Gastric Cancer. 2003;6(4):203-209.

33. Li Y, Yau A, Schaeffer D, Magliocco A, Gui X, Urbanski $\mathrm{S}$, Waghray R, et al. Colorectal glandular-neuroendocrine 
mixed tumor: pathologic spectrum and clinical implications. Am J Surg Pathol. 2011;35(3):413-425.

34. Bergsland EK, Roy R, Stephens P, Ross JS, Bailey M, Olshen A. Genomic profiling to distinguish poorly differentiated neuroendocrine carcinomas arising in different sites. ASCO Meet Abstr. 2016;34:4020.

35. de Mestier L, Cros J, Neuzillet C, Hentic O, Egal A, Muller N, Bouche O, et al. Digestive system mixed neuroendocrine-non-neuroendocrine neoplasms. Neuroendocrinology. 2017;105(4):412-425.
36. Albores-Saavedra J, Batich K, Hossain S, Henson DE, Schwartz AM. Carcinoid tumors and small-cell carcinomas of the gallbladder and extrahepatic bile ducts: a comparative study based on 221 cases from the Surveillance, Epidemiology, and End Results Program. Ann Diagn Pathol. 2009;13(6):378-383.

37. Kamboj M, Gandhi JS, Gupta G, Sharma A, Pasricha S, Mehta A, Chandragouda D, et al. Neuroendocrine carcinoma of gall bladder: a series of 19 cases with review of literature. J Gastrointest Cancer. 2015;46(4):356-364. 